Aus der chirurgischen Klinik zu Leiden. (Vorstand: Prof. Dr. J. H. Z a a i jer.)

\title{
Resultate der chirurgischen Behandlung der Appen- dicitis, beurteilt nach den letzten 1000 Fällen der chirurgischen Klinik zu Leiden.
}

Von Dr. W. F. Suermondt, Assistenzarzt der Klinik.

Mehr und mehr hat man während der letzten Jahre die große Bedeutung der Statistik bei der Beurteilung der verschiedenen Behandlungsmethoden anerkannt. Die Vergleichung der zahlreichen Nachuntersuchungen und Mortalitätsstatistiken aus verschiedenen Kliniken läßt uns den Wert unserer therapeutischen Maßregeln erkennen und uns ein Urteil fällen, wie unseren Patienten am besten geholfen wird.

Auf einer Sitzung des „Nederlandsch Natuur- en Geneeskundig Congres" in 1907 gehalten in Leiden, hielt $\mathrm{L}$ a $\mathrm{nz}$ einen Vortrag über die chirurgische Behandlung der Appendicitis, und seitdem ist man in Holland im allgemeinen der Ansicht, daß man das chirurgische Eingreifen der internen Behandlung vorziehen müsse. Über die Art der chirurgischen Behandlung ist man jedoch noch lange nicht einig. Hier stehen zwei Auffassungen einander gegenüber. Welche von ihnen sich auf die Dauer als die richtige herausstellen wird, hängt natürlich von den Resultaten ab.

Ich erlaube mir, auf die verschiedenen Meinungen der Chirurgen über die Behandlung gewisser Stadia von akuter Appendicitis etwas näher einzugehen.

Es ist wohl sicher, daß ein jeder den akuten Anfall im Frühstadium mit einer Radikaloperation zu bestreiten versucht; ebenso wird wohl kaum einer zögern, die akute allgemeine Peritonitis, verursacht durch Appendicitis, sobald wie möglich operativ zu behandeln, wobei er zugleich sich bemühen wird, die Ursache der 
Peritonitis, in diesem Falle den Appendix, weg zu nehmen, wenn das Allgemeinbefinden des Kranken ein derartiges Eingreifen noch rechtfertigt. Besteht indessen nur eine lokale Peritonitis in der Form eines umschriebenen abgekapselten Abszesses, dann wird die Sache etwas anders und laufen die Meinungen über die Behandlung auseinander.

Man hat die verschiedenen Stadia der akuten Appendicitis nach Zeit und Dauer der Krankheit in drei Gruppen eingeteilt: 1. das Frühstadium, 2. das Intermediärstadium von 3 bis 5 Tagen, 3. das Spätstadium nach 5 Tagen.

Diese Einteilung ist nach Wolff nicht so gut wie die folgende, die sich ausschließlich nach dem pathologisch-anatomischen Befund richtet. Er unterscheidet: I. die akute Appendicitis ohne jegliche Komplikationen. Der Prozeß hat sich auf dem Wurmfortsatz und seiner nächsten Umgebung beschränkt. 2. Appendicitis mit Abszeß; hier befindet sich eine abgekapselte Eiteranhäufung neben einer ganz freien Bauchhöhle, also eine lokale Peritonitis. 3. Appendicitis mit allgemeiner Peritonitis. 4. Chronische Appendicitis und Appendicitis im Intervallstadium. W olf $f$ hält diese Einteilung für besser wie die vorige, weil man alle Formen von der unkomplizierten Appendicitis bis zum AbszeB oder diffuser Peritonitis während aller drei Stadien antreffen kann. Es besteht nun ein großer Meinungsunterschied über die Behandlung der appendizitischen Abszesse. Im allge. meinen kennt man drei Methoden:

Die konservative Therapie, wobei man das lokale Infiltrat mit Ruhe behandelt und nur dann operativ eingreift, wenn Verschlimmerung des Zustandes eintritt, wobei man sich jedoch nur zum Öffnen des Abszesses beschränkt (Fromme, Krogius, Steiger, Hagen, Morris usw.).

Fromme schreitet nur zum Öffnen des Abszesses, wenn er gegen die vordere Bauchwand liegt. Kommt man zuerst in die freie Bauchhöhle, dann wird das Peritoneum wieder geschlossen und nach der Mitte abgeschoben, worauf der Abszeß von hinten geöffnet wird. Demgegenüber aber gibt es viele Chirurgen, die sich nicht scheuen, wenn Operation wegen Ausbreitung der Symptome notwendig geworden ist, den Abszeß quer durch die freie Bauchhöhle zu öffnen, wenn man nur dafür Sorge 
trägt, die Umgebung nach allen Seiten gut mit Gaze abzudecken, damit der Eiter sich nicht über die übrige Bauchhöhle verbreiten kann. Nach dieser Weise handelt man auch in der Klinik zu Leiden.

Durch Lanz wurde der konservative Standpunkt auf dem schon oben erwähnten Kongresse verteidigt. Seine Indikationen sind die folgenden:

1. Man verrichtet bei einem akuten Anfall so bald wie möglich die Frühoperation; dieselbe ausgeführt vor dem Ausbruch der allgemeinen eitrigen oder septischen Peritonitis ist ohne Gefahr.

2. Bei lokalisierter Entzündung (der klassischen Perityphlitis) ist während des Anfalls mit einem Eingriff zu warten. Kommt es zur Abszeßbildung, dann wird der Abszeß geöffnet längs des kürzesten Weges. Man muß sich indessen nur auf die Inzision beschränken und das Suchen nach dem Wurmfortsatz unbedingt unterlassen.

3. Beim Anfang der Peritonitis, am Tage der Perforation operiert $\mathrm{L}$ a $\mathrm{n} z$, entleert den Bauch und entfernt den Appendix. „Wie sehr ich auch bei der zirkumskripten, eitrigen Peritonitis die gleichzeitige Entfernung des Processus vermiformis bestreite, soll doch unbedingt bei diffuser Peritonitis der Ursprung der Eiterung entfernt werden, damit das Peritoneum nicht im Kampfe gegen immer aufs neue eindringende. Feinde unterliegen muß."

4. Im Spätstadium wartet L a n z unter sorgfältiger Kontrolle des Kranken mit absoluter Diät, absoluter Ruhe, Eisblase, kleinen Salz. klysmen und regelmäßigen subkutanen Infusionen ruhig ab. Bei dieser Behandlung sieht man oft einen Rückgang der Peritonitis, manchmal Bildung isolierter Abszesse und nur, wenn Verschlimme. rung des Zustandes auftritt, wird man zu einer Notoperation ge. zwungen sein.

5. Bei Kranken mit starkem Meteorismus, Zyanose, kaltem Schweiß, schlechtem Puls läßt er sich nicht zur Operation bewegen, beschränkt sich jedoch auf Salzwasserinfusionen, Spülen des Magens, Stimulantia und Kaffeeklysmata.

6. Wenn der Kranke von dem Anfall genesen ist und objektiv keine Reste davon mehr aufuweisen sind, ist der richtige Moment für die Operation „à froid" ‘da.

Eine Methode, die operativ schon etwas weiter geht, ist das prinzipielle Öffnen des Abszesses, sobald die Diagnose unumstößlich feststeht. In jedem Falle wird der AbszeB geöffnet, während man durch Palpation in der Abszeßhöhle sich davon überzeugt, ob der Wurmfortsatz frei liegt. Wenn er leicht zu entfernen ist, so tut man es, sobald es ohne Gefahr für die 
freie Bauchhöhle geschehen kann (Körte, Kümmel, Sonn e n burg usw.).

Die Verfechter der beiden oben erwähnten Methoden scheuen sich also bei einem AbszeB den Wurmfortsatz wegzunehmen, weil sie sich fürchten, von der abgekapselten Peritonitis eine diffuse zu machen. Sehr selten liegt aber der Appendix frei im Abszeß; meistens ist er in der Wand, so daß man beim Auspräparieren des entzündeten Organes in der Abszeßwand Risse macht, wodurch der Eiter sich in der übrigen Bauchhöhle ausbreiten kann. Außerdem läuft man ebenso Gefahr, Risse in den infiltrierten Wänden der umliegenden Darmschlingen zu machen, so daß man eine Darmfistel bekommen kann. Nach meiner Meinung darf man diese zweite Schwierigkeit nicht als Grund gegen die radikale Therapie anführen; diese Gefahr (der Darmfistel) droht uns ja bei jeder Abszeßöffnung, da wir niemals zuvor wissen können, ob der AbszeB nicht schon im Begriff war, in einen der umliegenden Därme durchzubrechen; es ist uns schon wiederholt vorgekommen, daß wir einen solchen Durchbruch durch unsere AbszeBinzision nicht vorbeugen konnten und kurze Zeit nach der Operation sehr unangenehm überrascht wurden, wenn Stuhl aus der Wunde kam.

Den zwei vorigen Methoden gegenuiber steht die radikale Behandlung: Öffnen des Abszesses und zu gleicher Zeit Entfernung des Wurmfortsatzes, eine Methode, die in Deutschland durch Rehn und in Frankreich durch Témo in verteidigt wird.

Hierunter folgen die Regeln, nach welchen in der Rehnschen Klinik der appendizitische Abszeß behandelt wird, zitiert aus der Arbeit von Wolff.

I. Jeder Fall von abgekapselter appendicitischer Eiterung wird, wie jede andere sichere Appendicitis, baldigst operiert, wenn er in die Klinik aufgenommen ist. Es wird weder in Intermediärstadium, noch im Spätstadium abgewartet.

2. Der AbszeB wird ohne Scheu durch die freie Bauchhöhle hin. durch und mit ausgiebiger Eröffnung derselben entleert. Es wird nicht ängstlich vermieden, die abschließende Verklebungen und Verwachsungen anzutasten, sie werden vielmehr so weit getrennt und gelöst, als es für eine genügende Freilegung und Übersichtlichkeit des infektiösen Prozesses, sowie für die Entfernung des Wurmfort. satzes notwendig ist. Der Eiter wird durch lokale Spülung mit Koch. salzlösung beseitigt. 
3. Der Wurmfortsatz wird, wenn irgend möglich, als die Ursache des Prozesses und eventueller Rezidive, bei der Operation selbst entfernt.

4. Durch Einlegen eines Gummidrainrohres in das kleine Becken wird für cine zweckmäßige Drainage dcr Bauchhöhle gesorgt, dic breit mit der Abszeßhöhle, dem Infektionsherd, in Verbindung steht. In das Abszeßbett wird, wenn es gangränöse oder jauchige Beläge zeigt, oder wenn das Cöcum morsch und phlegmonös crscheint, ein Streifen Jodoformgaze eingelegt.

5. Dic Bauchwunde wird in Etagen bis auf die Drainagestelle zugenäht.

$\Lambda$ uf diese Weise wird das appenzitische AbszeB in der Rehnschen Klinik behandelt, weil man die direkte Operation in jedem Stadium nicht für gefährlicher hält als das Abwarten; im Gegenteil, Rehn hält es für rationeller, den Kranken so bald wie möglich von der Eiteranhäufung in seinem Bauch zu befreien und wagt es nicht, sich auf die Möglichkeit der Resorption oder spontaner Perforation in Darm, Blase oder Vagina zu verlassen. Abgesehen von allgemeiner Peritonitis, verursacht durch Bersten des Abszesses in die freie Bauchhöhle oder durch sogenannte „Durchwanderung" von Bakterien, drohen beim Abwarten noch andere Gefahren, namentlich:

a) Sekundäre Eiteranhäufungen auf anderen Plitzen: subphrenische Abszesse, LungenabszeB, Pleuraempyem.

b) Thrombose der Mesenterialgefäße, mesenteriale Pyämie, Pyelophlebitis, foie appendiculaire.

c) Eiteransammlungen im retroperitonealen Gewebe mit tiefen Phlegmonen.

d) Starke Blutungen aus den großen Gefäßen.

Bei der radikalen Methode schaltet man diese Gefahren aus, weil aller Eiter weggespült wird und keine Eiterreste übrig bleiben, die die Ursache sekundärer Abszesse sein können. Wenn man nur den Abszeß öffnet, kann man kein Urteil abgeben über die übrige Bauchhöhle und übersieht leicht kleinere Abszesse, die manchmal abgekapselt neben dem größeren geöffneten Abszesse liegen. Das kann dem radikalen Operateur nicht passieren. $\mathrm{R}$ e h $\mathrm{n}$ und seine Anhänger raten diese radikale Therapie an, weil sie keine Infektion des freien Peritoneums fürchten und volles Vertrauen in dessen Resorptionsvermögen haben. Im Anfang des 
Anfalles tritt ein peritonealer Reizbarkeitszustand ein durch das Austreten von Giften und Bakterien aus dem kranken Appendix, wodurch die Widerstandsfähigkeit des Peritoneums gegen die Infektion erhöht wird. Es ist jetzt nur die Frage, wie lange man hierauf vertrauen darf. Laut $\mathrm{R}$ eh $\mathrm{n}$ dauert der Zustand der erhöhten Widerstandsfähigkeit einige Wochen, andere Autoren dagegen meinen nur einige Tage, nach ihnen dürfe man nur bis zum Ende des intermediären Stadiums radikal operieren. Ein Verfechter dieser Meinung ist Bertelsmann, dessen Auffas. sung erwähnt werden möge:

Das Peritoneum resorbiert nur im Anfang der Krankheit. Ist es jedoch einmal gereizt und dadurch in einen Zustand erhöhter Widerstandsfähigkeit versetzt, dann können keine Bakterien mehr ins Blut eindringen; daraus folgt, daß bei akuter Appendicitis die Verhältnisse zur Operation im Beginnstadium am günstigsten sind, da alle Abwehrkräfte des Peritoneums dann mobilisiert sind. Diesen $\mathrm{Zu}$ stand findet man auch noch im intermediären Stadium. Ganz anders wird es jedoch, wenn wir es mit einem abgegrenzten AbszeB zu tun haben, der schon von der übrigen Bauchhöhle so gut abgetrennt ist, daß er keine Fähigkeit mehr hat, hierauf einen Reiz auszuüben. (Klinisch ist dies auch ganz deutlich zu sehen, weil nur der scharfbegrenzte Abszeß druckschmerzlich ist, während der übrige Jeib ganz schmerzlos ist.)

Sind nun in einem solchen abgekapselten Abszeß noch virulente Bakterien, dann kann dieser Inhalt für die übrige Bauchhöhle gcfährlich werden, da dieselbe sich jetzt nicht mehr in einem Zustand erhöhter Reizbarkeit befindet. (Diese Auffassung wird auch durch klinische Beobachtungen bestätigt, denn die Perforation des Wurm. fortsatzes, selbst beim Anfang des Anfalles, ist lange nicht so gefähr. lich wie die Perforation eines schon einige Zeit bestehenden Abszesses. Man würde hier fast von einem anaphylaktischen Shock sprechen.)

Auf Grund dieser Erwägungen macht Bertelsmann im inter. mediären Stadium noch Appendektomie, während er bei älteren Abszessen nur drainiert und den Wurmfortsatz sitzen läßt, um die übrige Bauchhöhle nicht in Gefahr zu bringen. In seiner Klinik gelten folgende Regeln für die Behandlung alter Abszesse ( $\mathrm{K}$ a rl Gri e p):

1. Jeder Abszeß wird so bald wie nur möglich operiert.

2. Man beschränkt sich darauf, nur dic Abszeßhöhle zu entleeren, wobei natürlich sorgfältig Infektion der übrigen Bauchhöhle vermieden wird. Der Wurmfortsatz wird nur dann entfernt, wenn es ohne Gefahr für Infektion des Peritoneums möglich ist. Liegt der AbszeB nicht gegen die vordere Bauchwand an, dann wird nach sorgfältigem 
Tamponieren der Umgebung der Absze $B$ durch die freie Bauchhöhle geöffnet.

3. Prinzipiell wird der Appendix sekundär und am frühesten 3 Monate nach der Genesung des Abszesses entfernt. Man wartet ausdrïcklich so lange, da man dann die meiste Sicherhcit hat, da $B$ die Bauchwand p. p. genesen ist.

W olff behauptet, daB die sekundäre Ektomic durch die vielen Verwachsungen sehr schwierig sein kann. Laut Griep ist die Ope ration wohl beschwerlich, doch sicher nicht schwicriger als die primäre Ektomie beim Abszeß und nicht so gefährlich. Dabei vergleicht er die Statistiken aus beiden Kliniken: In der Klinik von Bertels. $\mathrm{m}$ a n n 62 Fälle mit $\mathrm{I}$ Todesfall $=1,6$ Proz

In der Klinik von Reh n 108 mit 12 Proz. Mortalität.

Die Bedenken, welche durch die Rehnsche Schule angeführt werden gegen die konservative Therapie, werden durch Griep folgenderweise widerlegt:

Gefahr für allgemeine Peritonitis, wenn der Absze $B$ berstet, be steht nicht, da Bertclsmann nicmals abwartet, doch den Abszeß sofort öffnet. Es könnte Gefahr sein für eine „Durchwanderungsperitonitis“; man soll jedoch erwägen, daß dies langsame „,Durch. wandern" die Abwehrkräfte des Peritoneums erhöht. Sicher ist dies doch weniger gefährlich wie eine plötzliche Überschwemmung wäh. rend der Operation. Sah doch Wolff bei scinen Fällen unter 25 Toten 7 mal eine progrediente Peritonitis. Weiter teilt W olf $\mathrm{f}$ noch mit, daß man manchmal in den ersten Tagen nach der Operation vorübergehende peritonitische Symptome findet: Darmparalyse, Erbrechen, Meteorismus, Défense musculaire. Manchmal waren diese Erscheinungen so stark, daß die Bauchwand aufplatztc, wenn Wolf $f$ nur mit Catgut geschlossen hatte. Darum gibt or den Rat, zwischen den Catgutnähten einige Seidennähte zur Unterstuitzung zu legen (eventuell Silber- oder Aluminiumdraht). Die dritte Gefahr einer konservativen Therapie würde das Rezidiv sein. Darum verrichtet man in der Klinik von Bertelsman $n$ denn auch prinzipiell cine sekundäre Ektomie; wird jetzt später ein Rezidiv kommen. bevor man zur Operation vorgeschritten, dann hat man durch die Adhäsionen des vorigen Anfalles und durch die Lage des Wurmfortsatzes aufs neue die meiste Aussicht auf einen abgekapsclten Abszeß.

Griep kommt jetzt zur Konklusion, daß die Operation in zwei Tempi nebst größerer Sicherheit und Ungefährlichkeit noch den Vorteil hat, daB:

I. Brüche vorkommen werden, da man mehr Aussicht auf Wundstörungen hat, wenn in einem Tempo operiert wird und

2. die Gefahr für lleus vermindert wird, weil man b:i der zweiten Operation in der Gelegenheit ist die schon geformten Adhäsionen zu Jösen. 
In Deutschland sind noch verschiedene andere Chirurgen Verfechter der radikalen Therapie.

Kreckez. B. operiert direkt jeden scharf umgrenzten Abszeß und nimmt den Appendix weg, wenn die Operation nicht gar zu schwer ist. Er hatte bei diesem Verfahren 5 Proz. Mortalität. $\mathrm{Er}$ beschränkt sich allein auf Inzision, wenn er unter ungünstigen Um. ständen operiert (z. B. auf dem Lande, bei sehr großen, schon lange bestehenden Abszessen, bei alten, schwachen Leuten und bei sehr starken Adhäsionen).

Eichhof hat 69 appendicitische Abszesse nach der Rehnschen Methode behandelt und hatte eine Mortalität von 4,6 Proz. Von diesen 69 Kranken bekamen 17 eine. Bauchwandinfektion, wovon 2 mit Nekrose der Fascie, 3 mal sah er eine spontane Darmfistel, I mal Ileus und 5 mal einen Narbenbruch.

$\mathrm{H}$ orst $\mathrm{R}$ i e d i g e r teilt uns mit, daß in der Klinik von $\mathrm{N}$ oe t. zel bei 98 radikal operierten Abszessen die Mortalität sich auf I, 2 Proz. belief, d. h. im Frühstadium 9,6 Proz., im Spätstadium I2,5 Proz.

I9I9 ist obige Frage in Paris in der Académie de Médecine zur Sprache gebracht.

Témo in, ein großer Vertreter der radikalen Therapie, operiert den akuten Anfall in jedem Stadium und hatte auf 2617 Radikaloperationen 83 Tote $=3,5$ Proz. Mortalität.

Die meisten französischen Chirurgen waren Anhänger einer mehr konservativeren Therapie. Hartmann z. B. operiert nicht, wenn ein scharf begrenztes, hartes Infiltrat zu fühlen ist, wartet erst die Resorption ab und verrichtet dann dic Appendektomie à froid.

Jalaguier operiert nur dann den Abszeß, wenn die allgemeinen Symptome sich verschlimmern, drainiert den Abszeß und nimmt den Wurmfortsatz nur mit, wenn er frei liegt. Darum seine Forderung den Kranken mit einem appendicitischen AbszeB in einer chirurgischen Klinik aufzunehmen und um sozusagen mit dem Messer in der Hand zu observieren, damit man augenblicklich eingreifen kann, wenn sich die Symptome verschlimmern, ,l'indication capitale n'est pas une question d'heures, mais une question de symptomes."

Auf diesem selben Standpunkt stehen auch Bazy, Tuffier und Walther. Auch in der Klinik zu Leiden wird nach den Prinzipien von J a la g u i er gehandelt. Unter denen, welche sich stark gegen eine radikale Behandlung sträuben, will ich noch Robert T. Morris nennen, der der Meinung zugetan ist, daß man viel mehr den natürlichen Abwehrkräften des Organismus im Streit gegen Infektionen überlassen darf. Morris operiert 
nur dann, wenn alle peritonitischen Erscheinungen verschwunden sind.

Nach der Mitteilung dieser so auseinander laufenden Meinungen über die Behandlung der appendizitischen Abszesse sei es mir erlaubt, die Methode unserer Klinik zu beschreiben. Vor allem gilt die Regel, im akuten Anfall so bald als möglich zu operieren. Wir ziehen keine scharfe Grenze zwischen einem Frühstadium oder intermediären Stadium. Während wir natürlich lieber in den ersten zweimal 24 Stunden operieren, wird ein Kranker, der am dritten Tage mit allen typischen Symptomen eines akuten Anfalles aufgenommen wird, auch dann noch operiert, wenn die Erkrankung sich anscheinend ausschließlich auf den Wurmfortsatz beschränkt hat (ich spreche hierbei nicht von der: Fällen, wobei schon peritoneale Reizerscheinungen bestehen).

Sobald jedoch ein Infiltrat zu fühlen ist, also eine lokale Peritonitis diagnostiziert ist, wird der Patient konservativ behandelt in der von Fowler angegebenen Lage mit Bettruhe, Eisblase und Diät. Ein Patient mit einem appendi. zitischen Infiltrat $m u B$ in einer chirurgischen Klinik verpflegt werden. Der Prozeß kann sich nämlich jetzt nach zwei Seiten hin entwickeln: entweder das. Infiltrat wird allmählich durch Resorption kleiner und verschwindet ganz, oder es geht in Erweichung über, und es bildet sich ein Abszeß. Hat sich einmal ein Abszeß geformt, so kann auch dieses ganz verschiedenartig auftreten. Im günstigsten Falle wird der Eiter teilweise resorbiert, teilweise eingetrocknet und auf diese Weise unschädlich gemacht; bei der später stattfindenden Operation sieht man nur einzelne Bröckelchen eingetrockneten Eiters, die Reste des früheren Abszesses. Auch kann der Abszeß verschwinden durch spontane Perforation in Darm, Harnblase oder Vagina, was indessen etwas weniger günstig ist, weil dann der Kranke manchmal nicht ganz beschwerdefrei ist, z. B. nach einer Perforation in die Harnblase, wenn eine Zystitis wegen Appendixfistel in der Harnblase besteht.

Der Zustand wird jedoch schlimmer, wenn der Abszeß sich nach der freien Bauchhöhle ausbreitet und die Gefahr für eine Perforation hierin droht. Geschieht dieses, dann kann nur eine unverzügliche Operation Hilfe bringen, und jede Minute der Ver- 
zögerung verringert die Möglichkeit eines Erfolges. Weil man niemals zuvor wissen kann, wie sich der Prozeß entwickeln wird, ist es sehr notwendig, den Patienten mit einem Infiltrat fortwährend unter chirurgischer Kontrolle zu halten, das heißt in einer Klinik, wo direkt operiert werden kann.

Die Diagnose ,,appendizitischer Abszeß“ ist für uns niemals eine Indikation zur sofortigen Operation. Wird ein Kranker mit dieser Diagnose aufgenommen, dann behandelt man ihn nach gründlicher Untersuchung mit Bettruhe, Diät, Fowlerscher Lage, Eisblase usw. Wir glauben zwar nicht, daß das Eis einen großen therapeutischen Effekt hat, aber die Eisblase ermahnt den Kranken, unbeweglich liegen zu bleiben. Der Patient wird fortwährend beobachtet, man achtet auf die Temperatur, den Puls, die abdominalen Symptome, Übelkeit usw.

Wenn wir durch Untersuchung per rectum, welche niemals unterlassen wird, festgestellt haben, daß der AbszeB vom Rektum aus nicht zu erreichen ist, dann ist doppelte Vorsicht geboten, da ein solch hochliegender Abszeß viel eher in die freie Bauchhöhle durchbricht als ein unten im kleinen Becken liegender. Sobald wir nun merken, daß der Abszeß die Neigung hat, sich nach oben und nach der Mitte zu auszubreiten und hohes Fieber, frequenter Puls, zunehmende Défense musculaire und Schmerz über den ganzen Bauch, Übelkeit und Erbrechen und eine kalte Nase uns als ebensoviele peritoneale Reizsymptome warnen, daß eine Perforation droht, dann werden wir nicht länger zögern und in Lokalanästhesie eine Inzision machen über die Mitte des Abszesses und die Abszeßhöhle drainieren. Dabei suchen wir nicht $\mathrm{nach}$ dem Wurmfortsatz. Wir beschränken uns bei diesen schwerkranken Patienten auf den einfachsten Eingriff und sorgen durch das Einlegen einiger Gummiröhrchen und Gazestreifen für gehörigen $A b f l u B$ des Eiters. Falls der AbszeB nicht gegen die vordere Bauchwand anliegt, wird nach dem Öffnen der Bauchhöhle und sorgfältigem Abdecken der Umgebung mit breiten Jodoformgazestreifen, die auch nach der Operation liegen bleiben, der Abszeß geöffnet und auf dieselbe Weise behandelt. Neulich hat Westerman auf der letzten Sitzung des „Nederlandsch Natuur- en Geneeskundig Congres" empfohlen, den Abszeß in der Fowlerschen Lage zu öffnen, um eine Verbreitung 
des Eiters durch die freie Bauchhöhle soviel wie möglich zu vermeiden, ein Rat, den man in der Zukunft zu Herzen nehmen wird. Nach der Operation wird der Kranke in der Fowlerschen Lage so wie zuvor behandelt. Im Laufe der folgenden Tage werden allmählich die Tampons entfernt, die Röhrchen verdünnt, verkürzt und schließlich ganz weggelassen; nachdem die Abszeßinzision sich geschlossen hat, was meistens ungefähr 3 bis 4 Wochen dauert, wird der Patient entlassen, um nach 8 oder Io Wochen à froid von seinem Wurmfortsatz befreit zu werden. Wenn nach der Aufnahme eines Kranken mit einem appendizitischen Infiltrat keine peritonealen Reizsymptome gefunden werden oder dieselben langsam zurückgehen, dann operieren wir nicht; nicht so ganz selten werden wir dann durch eine spontane Perforation in ein Intestinum überrascht, wonach öfters sofort alle Symptome verschwinden, die Temperatur normal wird und von einem Infiltrat nichts mehr zu finden ist. Der Patient mit einem konservativ behandelten Infiltrat bleibt in der Klinik, bis die Geschwulst ganz verschwunden ist und wird dann 8 bis Io Wochen später à froid operiert.

Obgleich ich später ausführlich unsere Resultate besprechen werde, möchte ich doch jetzt schon mitteilen, daß nach oben genannten Prinzipien durch uns 182 Kranke mit appendizitischem Infiltrat oder Abszeß behandelt sind mit o Proz. Mortali. tät, so daß meines Erachtens für uns vorläufig keine Veranlas. sung besteht, vion unserem konservativen Standpunkte abzu. weichen.

Zum Schluß bleibt mir noch übrig, den akuten Anfall mit allgemeinen peritonealen Erscheinungen zu besprechen, wobei das Peritoneum über eine größere Ausgestrecktheit krankhaft verändert ist. Jetzt ist kein Infiltrat zu fühlen, da das Peritoneum keine Kraft hat, den Prozeß durch Abkapselung einzuschränken. Hier warten wir nicht erst ab, greifen direkt operativ ein und versuchen den Wurmfortsatz, die Ursache des Ưbels, wegzunehmen; denn hier, wo keine Spur einer Begrenzung der Ent. zündung besteht und das Bauchfell bis weit in der Umgebung angegriffen ist, braucht man das Freimachen des Wurmfortsatzes nicht zu befürchten. Die vorschreitende Peritonitis, welche sichtlich keine Neigung hat sich abzugrenzen gegenüber der 
übrigen Bauchhöhle und in vielen Fällen verursacht wird durch einc Perforation des Blinddarmes, kann nur günstig beeinflußt werden durch die Exstirpation der Infektionsursache, von wo aus fortwährend infektiöses Material in die Bauchhöhle strömt. Man darf hierbei aber nicht zu weit gehen. Natürlich gehört zu einer Appendektomie unter obigen Umständen einige Widerstandsfähigkeit des Kranken. Das Entfernen des kranken Organes wird durch die allgemeine Bauchspannung und Schmerzen höchstwahrscheinlich nicht unter Lokalanästhesie gelingen, so daß man wohl genötigt ist, Allgemeinnarkose zu geben. Man kann es nicht gut verantworten, wenn man bei einem schwerkranken Patienten, der an einer allgemeinen Peritonitis leidet und beinahe ohne Puls und in sehr schlechtem Zustand aufgenommen wird, prinzipiell, weil keine Abkapselung da ist, zur Appendektomie schreitet! In solchen Fällen, wo man von einem einiger. maßen größeren Eingriff keinen Erfolg mehr erwarten darf, muß man sich damit zufrieden stellen durch das Anlegen von Drainieröffnungen in den beiden Flanken dem Eiter aus der Bauchhöhle Abfluß zu verschaffen.

Für die Appendektomie à froid gelten die folgenden Indikationen:

a) Wenn ein Infiltrat in der Klinik spontan resorbiert oder in ein Intestinum perforiert ist; 8 bis 10 Wochen nach dem Ver. schwinden des Infiltrates wird die Appendektomie ausgeführt.

b) Wenn man bei einem AbszeB wegen Ausbreitung der Symptome drainiert hat; 8 bis Io Wochen nach Genesung der Inzision wird die sekundäre Appendektomie verrichtet.

c) Wenn durch diesen oder jenen Umstand der Kranke während des akuten Anfalles nicht in einer Klinik aufgenommen war, doch konservativ behandelt wurde; in diesen Fällen wird die Indikation zur Appendektomie gestellt durch den Arzt, der den Anfall behandelt hat.

Nachdem ich jetzt die Indikationen zur Operation in den verschiedenen Stadien der Appendicitis besprochen habe, will ich nun näher auf die Technik eingehen.

Was die Anästhesie anbetrifft, darf ich wohl behaupten, daß im Gegensatz zu früheren Jahren gegenwärtig praktisch keine einzige Appendektomie mehr unter Narkose angefangen wird. 
In vieler Fällen gelingt es, die ganze Operation unter Lokalanästhesie zu vollenden; nur wenn der Wurmfortsatz sehr verwachsen ist mit seiner Umgebung, z. B. bei einer sekundären Appendektomie nach früherer AbszeBdrainage muß die Lokalanästhesie durch kurzdauernde Chloräthyläthernarkose unterstützt werden.

Beim üffnen des Bauches wählen wir in einfachen Fällen gerne die Inzision nach Mac Burney, in schweren Fällen den Lennanderschen „Kulissenschnitt“. Der „Wechselschnitt“, wobei alle Schichten der Bauchwand in ihrer Faserrichtung gespaltet werden, hat den großen Vorteil, die Bauchwand nur wenig von ihrer Festigkeit $z u$ berauben, so daß man Narbenbrüche nach dieser Inzision beinahe niemals sieht. Ein Nachteil dieser Schnittführung dagegen ist, daß3 man nur einen kleinen Teil der Bauchhöhle überblicken kann, so daß man bei einer schweren Appendektomie viel vorteilhafter den „Lennander-Schnitt“ anwenden kann, der uns einen viel besseren Einblick in die Bauchhöhle ge währt, dagegen aber mehr Möglichkeit zu einer späteren Bruchformung bietet, da man öfters gezwungen ist, die zuführenden Nerven des M. rectus zu durchschneiden, wodurch ein Teil des Muskels paralytisch wird. Doch wird der "Lennander-Schnitt" in unserer Klinik gern angewendet, besonders wenn man eine schwierige Operation erwarten darf oder wenn man aus bestimm. ten Gründen die Narkose soviel wie möglich vermeiden will. Durch den viel größeren Zugang, den diese Schnittführung uns bietet, ist es leicht begreiflich, daß wir jetzt mehr Aussicht haben, die Operation unter Lokalanästhesie zu vollenden.

Nach D u ra nd muß der Schnitt bei der Intervalloperation zwei Bedingungen genügen; erstens muß er einen guten Überblick über die Gegend des Wurmfortsatzes geben und zwcitens zu ciner so gering möglichen Beschädigung der Bauchwand Veranlassung gcben. D u • rand findet den Schnitt nach Mac Burney am besten, obwohl dieser nicht ganz der ersten Anforderung entspricht; deshalb soll man ihn nur dann anwenden, wenn die Diagnose feststeht und die Entzuindungserscheinungen ganz zur Ruhe gekommen sind.

Die Technik der Appendektomie gibt keine Veranlassung zu besonderen Bemerkungen; nur möchte ich noch erwähnen, daß3 in den Fällen, wo die Basis des Wurmfortsatzes infiltriert ist, dieser zwischen zwei elastischen I armklemmen mit dem Thermo- 
kauter durchtrennt wird, da hier beim Anlegen der Darmquetsche, die gewöhnlich angewendet wird, der Blinddarm leicht bersten kann, da die infiltrierte Wand sich nicht genügend zerquetschen läßt. In Übereinstimmung mit der Auffassung von Wolff sind wir sparsam mit der Resektion des entzündeten Netzes, erstens weil es nach der Entfernung der krankmachenden Ursache meistens spontan ausheilt, und zweitens, weil einmal Netzunterbindungen auf embolischem Wege Magenblutungen verursacht haben. Ist ein Teil des Netzes gangränös oder nekrotisch, dann wird es reseziert, damit das tote Stiuck keine Veranlassung zu Nacheiterungen geben kann.

Es ist eine wichtige Frage, wie man nach einer Appendektomie den Eiter in der Bauchhöhle entfernen und die Bauchhöhle drainieren soll. Es bestehen zwei Methoden:

e rste ns die sogenannte "trockne Methode", wobei man den Eiter soviel wie möglich wegfegt, soweit dies die Größe der Inzision zuläßt. Durch die Gegner dieser Methode wird angeführt, $\mathrm{da} ß$ man die Bauchhöhle lange nicht so gründlich reinigen kann als bei der anderen Methode, und daß man darum auch bei der trocknen Methode viel mehr Restabszesse sieht als

zweitens bei der "Spülmethode", wabei mittels einer in den Peritonealraum eingeführten Röhre so lange mit physiologischer Kochsalzlösung in allen Richtungen gespült wird, bis die Flüssigkeit ganz klar ausfließt. Diese Methode wirkt sicher gründlicher und schneller wie die erstgenannte; hat jedoch den Nachteil, daß man beim Ausspülen Bakterien dahin führt, wo sie vorher noch nicht waren.

Sohn teilt mit, daB Rotter bei $2 \mathrm{I}$ trocknen Behandlungen I 2 intra-abdominale Restabszesse sah, während $R$ eh n unter 449 ausgespülten Fällen 12 Restabszesse hatte. $\mathrm{K} \ddot{\mathrm{r} t \text { e hatte bei gespülten }}$ Fällen eine Mortalität von 40 Proz. und bei ausgefegten Fällen 42,2 Proz., wobei noch die Bemerkung gemacht wurde, daß Körte die schweren Fälle immer spülte.

Nachdem man so gut wie nur möglich den Eiter aus der Bauchhöhle entfernt hat, kommt die Frage, ob man den Bauch gleich schließen oder drainieren muß und wie letzteres am besten getan wird. Rotter z. B. schließt die Wunde sofort, da er meint, daß eine genügende Drainage der Bauchhöhle unmöglich 
ist und das sofortige Schließen eine schnellere Genesung bewirkt. Die meisten Chirurgen aber behaupten, daß es besser ist zu drainieren; dazu bringt man den Kranken soviel wie möglich in die Fowlersche Lage, macht das Cavum Douglasi zum tiefsten Punkte der Bauchhöhle und führt dann die Drains bis in das kleine Becken. Wo lf $f$ gibt den Rat, den Drain bis auf den Boden des kleinen Beckens zu bringen; dorthin sinkt der Eiter und steigt dann in das Rohr auf. Breitet sich der AbszeB mehr nach oben zu aus, dann führt er ein zweites Rohr dorthin oder er legt einen Gazestreifen auf das Appendixbett, eventuell mit einem Rohr dabei. Der Gazestreifen darf nicht auf der Darmnaht liegen, da diese sonst insuffizient wird. Weiter soll man darauf acht geben, daß der Drain nicht zu lange auf derselben Stelle liegen bleibt, damit kein Decubitus der Wand eines Darmes oder Blutgefäßes veranlaßt wird.

Deswegen rät Wolff, die Röhre während der ersten Tage regelmäßig zu bewegen. Nach dem dritten Tage verwechselt und verdünnt er die Drains und den siebenten oder achten Tag läßt er sie ganz weg; den fünften oder sechsten Tag wird der Gazestreifen herausgenommen, es sei denn, daß man schon eher vermutet, daß Retention unter dem Tampon ist, in welchem Fall es anzuraten ist, den Gazestreifen herauszunehmen und ihn durch ein Rohr zu ersetzen. Meistens wird das Rohr von einer rechtsseitigen, pararektalen Inzision aus bis in das kleine Becken eingeführt. In der Klinik von $L$ äw e n hat man jedoch Bedenken gegen obiges Verfahren; dort drainiert man lieber von einer aparten suprasymphysänen öffnung aus, crstens, weil der Weg zum Cavum Douglasi dann kürzer ist, und zweitens, weil das Rohr dann nicht über die großen Beckengefäße hinläuft und dadurch Decubitus eines Gefäßes soviel wie möglich vermindert wird. Durch Sohn wird dic Technik dabei wie folgt beschrieben: Nachdem aus einer rechtsseitigen, pararektalen Inzision der Wurmfortsatz entfernt ist, wird gerade über der Symphysis eine ungefähr $3 \mathrm{~cm}$ lange mediane Laparotomie gemacht. (Es ist notwendig, die Harnblase vor der Operation zu entleeren.) Von hier aus wird ein Katheter bis in das klcine Becken geführt und nachdem der Pat. in eine halbsitzende Stellung gebracht ist, wird jetzt so lange gespült, bis die Flüssigkeit klar abläuft. Der Abfluß des Wassers wird durch jedesmaliges Sinkenlassen des Clastrichters geregelt, so daß keine Flüssigkeit zurückbleiben kann. Danach wird das Gummirohr durch diese kleine mediane Inzision bis ins Cavum Douglasi geschoben. Bej 67 Fällen von Peritonitis nach Appendicitis, worunter 25 im Spät- 
stadium, die alle nach dieser Methode behandelt wurden, hatte L äw e n 28,3 Proz. Mortalität.

Verklebungen von Darmschlingen um das Rohr herum befördern das Entstehen von Verwachsungen und postoperativem Ileus. Die Furcht hiervor und vor Arrosion eines Blutgefäßes waren Gründe für Wilms, den Rat zu erteilen, das Cavum Douglasi von unten aus zu drainieren, und zwar beim Mann vom Rektum aus, bei der Frau von der Vagina aus. Die Methode $\mathrm{W}$ ilms wird durch $\mathrm{W}$ olf $\mathrm{f}$ abgelehnt, weil man dafür eine neue Inzision machen muß, wobei gesunde Bindegewebespalten geöffnet werden, so daß leicht eine phlegmonöse Entzündung des Beckens entstehen kann.

Nach Sohn hat die von Wilms angegebene Drainage die folgenden Nachteile: I. Ein schr bakterienreiches Gebiet wird mit der Bauchhöhle in Verbindung gebracht. 2. Es besteht Gefahr, die Harnblase zu beschädigen. 3. Die Drainage nach unten verlängert die Operation und macht sie komplizierter.

Ein einziges Mal hält Soh n die Drainage nach unten für nütz. lich, wenn nämlich von oben aus ungenügend drainiert ist; in diesem Fall sind schon so viele Verklebungen da, daß die sekundäre Drainage nach unten das Rektum nicht in direkter Verbindung mit der Bauchhöhle bringt.

In unserer Klinik wird die Drainage nach W ilms auch beinahe niemals angewendet, da ein $A b s z e B$ im kleinen Becken, der schon vom Rektum oder der Vagina aus zu fühlen ist, in den meisten Fällen spontan perforiert. Als Material zum Drainieren gebraucht man im allgemeinen Gummi- oder Glasrohre und Jodoformgazestreifen. Man ist jetzt ganz einig darüber, daß Gazestreifen allein für die Drainage nicht zweckmäßig sind, da sie direkt Anleitung zu Verklebungen geben; deshalb gebraucht man die Gazestreifen als Drainiermaterial nur in Verbindung mit Röhren.

Graser hat den Gebrauch der Dreesmannschen Drainierröhrchen warm empfohlen; in dem Glasrohr wird cin schmaler Gazestreifen geschoben, der den Eiter aus der Tiefe aufsaugt; die Gazestreifen können erneuert werden, während das Rohr liegen bleibt. Graser sieht gerade hierin den großen Vorteil dieser Röhrchen, da beim Wechseln der Drains die Darmschlingen sich manchmal so schnell gegeneinander anlegen, daB man den schon geformten Weg, wo die Drainage entlang lief, nicht mehr zurückfinden kann. Beim 
Gebrauch der Dreesmannschen Drains wird man dieser Schwierig. keit enthoben.

In Leiden wird bei einer Appendektomie, wobei sich Eiter in der freien Peritonealhöhle befindet, nach der ,trocknen Methode" gehandelt, weil man das Spülen der entzündeten Bauchhöhle für den Kranken zu angreifend findet; man beschränkt sich darum auf das Wegtupfen des Eiters von der Laparotomiewunde aus. Danach wird ein langes Gummirohr, worin seitliche Öffnungen geschnitten sind, bis auf den Boden des kleinen Beckens gebracht; eventuell legt man noch ein zweites Rohr nach der Stelle hin, von wo der Wurmfortsatz entfernt wurde; Jodoform. gazestreifen werden in Übereinstimmung mit der Auffassung Läwens nach einer Appendektomie nur selten angewendet; dann und wann wird in einem geöffneten Abszeß neben dem Gummirohr auch ein Gazestreifen eingeführt; nämlich wenn man eine große unregelmäßig geformte Abszeßhöhle findet, wobei man bestrebt ist diese so schnell wie möglich zu verkleinern; die Gazestreifen befördern die Entwicklung der Granulationen.

Unter den von mir gesammelten 1000 Fällen von operativ behandelter Appendicitis waren 227 mit Drainage nach der Ektomie. Bei 43 Kranken wurde während der Nachbehandlung ein Restabszel., entdeckt, und zwar:

$\begin{array}{lr}\text { subphrenisch } & 3 \mathrm{mal}, \\ \text { im Cavum Douglasi } & 23, ., \\ \text { links unten im Bauch } & 8, . \\ \text { rechts ", " ", } & 9, .\end{array}$

Von diesen Restabszessen sind 20 spontan perforiert in einen Darm, Rektum oder Vagina; 6 sind resorbiert und i 7 mußten operativ entleert werden. Wir waren also gezwungen, bei 227 drainierten Fällen I 7 mal sekundär einzugreifen $=$ in 7.5 Proz. der Fälle.

Nachdem die Drainage angelegt ist, werden die Schichten der Bauchwand geschlossen bis auf eine kleine Öffnung, wo das Rohr ausmündet. Z a a ijer schließt in solchen Fällen bei einer pararektalen Inzision das Peritoneum mit geknöpften Catgutnähten. die Fascie und die Haut aber mit geknöpften Seidennähten in einer Schicht. Beim Wechselschnitt, wobei dieses Verfahren nicht gut möglich ist, schließt er alle Schichten mit geknöpften Catgut- 
nähten. W o lf $f$ sah einmal, als der Leib nach der Operation durch Darmparalyse stark aufgetrieben war, ein Aufspringen der Naht und Prolaps des Eingeweides bei Verwendung von Catgut. Hatte er nur Seidennähte gebraucht, dann sah er langdauernde Fadenfistem. Deshalb rät er an, zwar mit Catgut zu schließen, aber dazwischen einige seidene Stütznähte anzulegen.

Das sorgfältige Schließen der Bauchwand kürzt die Dauer der Genesung ab und vermindert das Entstehen der Narbenbrüche; dem gegenüber steht die größere Gefahr der Bauchwandinfektion.

Wolff teilt mit, daß nur bei der Hälfte seiner Kranken die Bauchwand ,per primam intentionem“ genas, bei der andcren Hälfte hingegen die Wunde infiziert war. Er gibt folgende Zahlen:

$\begin{array}{ll}\text { Baucliwand per primam genesen } & 5 \text { I, } 5 \text { Proz. } \\ \text { subkutane Eiterung } & 34,9, " \\ \text { tiefe } " & 13,6, .\end{array}$

(Diese Zahlen beziehen sich nur auf Appendektomien mit abgekapselten Abszessen, mit andern Worten Fälle, die sehr geeignet sind für das Auftreten einer Infektion.)

Es waren also meistens subkutane Eiterungen, wobei man nur die Hautnaht öffnen mußte. Das subkutane Fett scheint sehr empfindlich zu sein. Wartet man zu lange mit dem Ausnehmen der Hautnähte, so läuft man Gefahr, daß die Entzündung sich über die tieferen Schichten ausbreitet und die Fascie nekrotisch wird. Wenn der Kranke den vierten oder fünften Tag anfängt zu fiebern und übrigens die Bauchhöhle ruhig ist, dann droht nach $\mathrm{W}$ olf $\mathrm{f}$ eine Bauchwandinfektion; in diesem Fall gibt er den Rat, die Hautnähte zu entfernen und die Wunde zu öffnen, da er lieber einige Nähte unnötig aufopfert, anstatt zu spät zu öffnen. Weiter ist es nach seiner Meinung ratsam alle Blutungen sehr exakt zu stillen, da jedes Hämatom natürlich ein fruchtbarer Nährboden für Bakterien ist.

Da B ertelsmann den Wurmfortsatzstumpf als die Ursache der Bauchwandentzündung betrachtet, durchtrennt er den Wurmfortsatz mit dem Thermokauter, da dies zweifellos aseptischer ist wie das Durchklemmen.

Eisendraht geht so weit, daß er beim Schließen zwischen den verschiedenen Schichten der Bauchwand jedesmal drainiert, und zwar legt er cin Rohr bis auf das Peritoneum, ein zweites zwischen den Muskeln und ein drittes unter der Haut.

Andere Chirurgen sind der Meinung, da $\beta$ man die Indikation zum primären Verschluß des Bauches viel weiter ausbreiten kann.

$\mathrm{R} u 1$ is on $z$. B, schließt ohne Drainage, wenn die krankhaften Veränderungen sich auf den Wurmfortsatz beschränken und eine 
geringe Menge klares, nicht stinkendes Exsudat vorhanden ist. Auch bei lokaler Peritonitis, die sich nur auf die Umgebung des Wurmfortsatzcs ausgebreitet hat, kann man nach seiner Meinung von der Drainage absehen. Ist jedoch das Bauchfell über eine große Ausdehnung mit fibrinös-eiterigem Exsudat bedeckt oder wenn das Exsudat stinkt, rät er an zu drainieren.

Noch weiter geht $Z$ ur Verth, der bei Frühoperationen den Bauch ohne Drainage schließt, auch wenn sich trübe oder eiterige Flüssigkeit in der Bauchhöhle befindet, ja selbst wenn ein perforierter Wurmfortsatz entfernt wurde.

Ich habe bei 955 Appendektomien 53 mal eine Bauchwandinfektion gefunden: $=5,5$ Proz., wie folgt verteilt:

I. 23 mal nach primärem Verschluß der Bauchwand, trotzdem Eiter oder Fibrin in der Umgebung des entzündeten Wurmfortsatzes war.

2. 3 mal nach primärem Verschluß, wobei im Protokoll vermeldet wurde, daß ein eiteriges Appendixbett in der Bauchhöhle zurückblieb.

3. 12 mal bei 227 drainierten Ektomien.

4. 15 mal, wobei ich in der Krankengeschichte oder im Operationsprotokoll keine Ursache für das Auftreten der Infektion finden konnte. Aus dieser Statistik sehen wir also, daß wir mehr Bauchwandinfektionen hatten in den Fällen, wo die Wunde direkt ganz geschlossen wurde, trotzdem infektiöses Material in der Tiefe zurückgelassen wurde. Die Zahl der Wundstörungen bei drainierten Fällen ist dagegen viel geringer, obschon hier natürlich viel ungünstigere Umstände vorlagen. Daraus folgt meines Erachtens, daß man in dubiösen Fällen nicht zu sparsam mit dem Drainieren sein soll, um Bauchwandinfektionen zu verhüten, zumal man durch die Drainage die Dauer der Nachbehandlung nur um einige Tage verlängert, eine Bauchwandinfektion dagegen eine erhebliche Verzögerung gibt.

Hat sich die Entzündung schon auf das Cöcum und die umgebenden Darmschlingen ausgebreitet, so ist das Auftreten der spontanen Darmfisteln eine unangenehme Komplikation nach Ektomien. Je eher also der Anfall operiert wird, desto weniger Gefahr zur Fistelbildung. Umgekehrt werden Chirurgen, die den Abszeß radikal operieren, also in einem Terrain arbeiten, 
wo die Entzündung schon lange die umgebenden Darmschlingen angegriffen hat, Fisteln nach der Operation eher sehen.

Wolff z. B. sah sie bei 8,8 Proz. der radikal behandelten Abszesse. Er glaubt, daß die Fistel durch die phlegmonöse Veränderung des Cöcums verursacht wird, wodurch die Nähte über dem Wurmfortsatzstumpf sich lockern. Ist schon während der Operation eine Perforation in einem Darm entstanden, dann übernäht er das Loch; bei einer drohenden Perforation wird prophylaktisch Netz auf der schwachen Stelle genäht.

Wer den Abszeß konservativ behandelt, wird die Kotfistel wohl umgehen können, wenn er nicht einzugreifen braucht. Wenn er jedoch durch Verschlimmerung der Symptome gezwungen ist den $A b s z e B$ zu öffnen, dann läuft er ebenfalls Gefahr, eine spontane Darmfistel zu bekommen. Der Abszeß ist vielleicht gerade im Begriffe, in den Darm zu perforieren. Nun ist glücklicherweise das Auftreten einer spontanen Darmfistel nicht allzusehr beunruhigend, da sie sich in vielen Fällen wieder von selbst schließt (Bertelsmann, Wolff).

Unter unseren Fällen fand ich 4 mal nach einer Appendektomie und ro mal nach einer AbszeBöffnung eine spontane Fistel; von diesen 14 haben sich Io spontan geschlossen ohne jegliche Hilfe; 3 wurden operativ geschlossen und I ist an Inanition zu grunde gegangen, trotzdem eine Colonfistel angelegt war, um den aus der Fistel strömenden Darminhalt hier wieder einzuspritzen.

Daneben kann die Abszeßöffnung noch eine andere Schwierigkeit mit sich bringen; manchmal will sich die kleine Wunde nicht schließen und fließt fortwährend eine geringe Menge schleimiger oder eiteriger Flüssigkeit ab; meistens hat man es dann mit einer Appendixfistel zu tun oder mit einem frei in der Bauchhöhle liegenden Kotstein. Wir sahen 4 mal nach einer Abszeßöffnung eine Appendixfistel und 3 mal eine Fistel, verursacht durch einen frei liegenden Stein. Diese Komplikation kann natürlich auch nach einer Ektomie vorkommen, wenn bei der Operation der Stein neben dem perforierten Wurmfortsatz übersehen wurde.

Griep teilt mit, daß man bei retroperitonealen Eiterungen langdauernde Fisteln finden kann, ohne da $B$ man es zu tun hat mit ciner Appendixfistel oder freiliegendem Kotstein. Öfnen und Aus- 
kratzen der Abszeßhöhle bringt Genesung. Einen solchen Fall sah auch $Z$ a a ijer cinmal, wobei nach der Abszeßöffnung eine Fistel übrig blieb, die, wie man sich bei der sekundären Ektomie überzeugen konnte, nicht abhängig war von den zwci obigen Ursachen; die Fistel wurde durch einen retroperitonealen AbszeB erhalten, dèr gegen die Wirbelsäule lag.

Nochmals möchte ich den Wert der Enterostomie betonen, die solch gute Resultate beim Kampf gegen den paralytischen lleus geben kann. Nach der Operation trachtet man die Darmperistaltik so bald wie möglich anzuregen, da Hemmung derselben Fäulnis des Darminhaltes verursacht, wodurch der Kranke vergiftet wird. Gelingt dieses nicht verhältnismäßig schnell mit den gewöhnlich gebrauchten Mitteln und wird der Bauch mehr und mehr aufgetrieben, dann wartet $\mathrm{Z}$ a a ijer nicht länger, da die Darmschlingen sonst so stark überdehnt werden, daß man kaum mehr auf eine Genesung hoffen darf. Er legt dann schnell unter Lokalanästhesie eine oder mehrere Darmfisteln an, eventuell kombiniert mit einer Gastrostomie, wenn der Kranke durch fortwährendes Erbrechen gequält wird. Auf diese Weise hat er schon öfters einen paralytischen Ileus geheilt.

Da eine Enterostomie für einen schwerkranken Pat. immerhin ainen großen Eingriff bedeutet und obendrein nach der Operation der Darm manchmal nur teilweise entleert wird, hat West er m a n $n$ in 1920 den Rat erteilt den Magen permanent durch eine Sonde zu drainieren, die durch die Nase bis in den Magen geführt wird. Der Kranke wird oft durch lästiges Erbrechen geplagt; durch Aushebern des Magens kann man nur zeitweilig Linderung verschaffen, man muß dieses Aushebern also jedesmal wiederholen, was für viele Kranken sehr unangenehm ist. West e r man n s' permanente Nasendrainage macht es uns leichter, während es obendrein für den Kranken noch den Vorteil hat, daß er so viel trinken kann, wie er nur will. Da durch das fortwährende Aushebern nur wenig Flüssigkeit aufgenommen wird, muß man auf anderen Wegen (Klysmata usw.) für genügende Wascerzufuhr Sorge tragen.

Auch Stutzin hielt das frühzeitige Anlegen eincr Darmfistel zur Bestrcitung des Meteorismus boi Peritonitis für sehr wichtig.

Sohn rät cbenfalls an, nicht zu lange mit Enterostomie zu wart'n, fügt jedoch gleicherzeit hinzu, es auch nicht zu früh zu tun, da (ine Dünndarmfistel immerhin keine Kleinigkeit ist und auf die Dauer zur Inanition führen kann, selbst wenn die Öffnung in der Nähe des Cöcums angelegt ist. Außerdem soll zum Verschluß der Fistel stets eine zweite Operation nötig scin. 
Diese beiden Schwierigkeiten dürfen jedoch in der Klinik zu Leiden bei der Beurteilung des Wertes der Enterostomie keine Rolle spielen; zum Beweise gebe ich folgende Zahlen:

Bei 3I Patienten wurden wegen paralytischen Ileus eine oder mehrere Darmfisteln angelegt; 22 mal mit Erfolg, 9 mal ohne Erfolg. Von diesen mußten später 3 operativ geschlossen werden; in den meisten Fällen war die Fistel einen Tag nach der Herausnahme des Rohres schon ganz geschlossen. Zweifellos werden diese günstigen Resultate durch die Anwendung der Witzelschen Schrägfistel befördert, welche sich nach Entfernung des Rohres von selbst schließt. An Inanition durch zu starkes Ausfließen von Darminhalt aus einer von uns angelegten Darmfistel haben wir noch niemals einen Kranken verloren. In den zwei Fällen, in denen sehr viel Flüssigkeit aus der Fistel strömte, haben wir später eine Kolostomie angelegt und in die Colonfistel wieder eingespritzt, was aus der ersten Fistel abfloß; auf diese Weise gelang es, die Kranken in gutem Ernährungszustand zu erhalten.

Zum Schluß möchte ich noch erwähnen, daß wir bei der Bestreitung der Darmparalyse von Injektionen mit Hormonal oder anderen, die Peristaltik anregenden Mitteln wenig Erfolg sahen.

Statistik der letzten 1000 operativ behandelten Fälle von Appendicitis während der Jahre 1905-1920. Es handelte sich um 513 Frauen und 487 Männer.

A. Männer.

Alter
1-5 Jahr :

Akut Gestorben Chronisch Gestorben 2

$\begin{array}{rrr}5 & 6 & \\ 4 & 18 & \\ 5 & 24 & \\ 2 & 26 & 1 \\ 2 & 22 & \\ 1 & 19 & \\ 2 & 12 & \\ 2 & 4 & \\ & 6 & \\ 3 & 2 & \end{array}$




\begin{tabular}{|c|c|c|c|c|}
\hline Alter & & Akut & Gesturben Chronisch & Gestorben \\
\hline $56-60$ Jahr: & 9 & 5 & 4 & \\
\hline $61-65 \quad, \quad:$ & 6 & 5 & 1 & \\
\hline $66-70 \quad, .:$ & I & 1 & & \\
\hline $71-75 \quad, \quad:$ & 2 & 2 & 1 & \\
\hline unbekannt: & 16 & l I & 5 & \\
\hline Total: & 487 & 338 & 149 & I \\
\hline
\end{tabular}

Von den akuten Fällen sind operiert:

unter Lokalanästhesie

anfangs Lokalanästhesie, später Narkose

in Narkose

\begin{tabular}{r|r}
93 & Ektomien \\
125 & Ek
\end{tabular}

Appendicitis im Intervallstadium und chronische Appendicitis: unter Lokalanästhesic

anfangs Lokalanästhesic, später Narkose

in Narkose

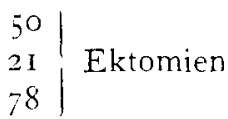

Nur Abszeßöffnung und später nicht zurückgekommen für

Radikaloperation

I I

wovon unter Lokalanästhesie

in Narkose

Drainage der Bauchhöhle bei diffuser Peritonitis ohne Appendektomie: I6, alle unter Lokalanästhesie.

Auf 338 akuten Fällen waren 29 Tote $=8,55$ Proz., und zwar: Peritonitis

periappendicitische Versenkungsabszesse

Perforation eines subphrenischen Restabszesses in der Pleura 1 Ileus

Auf 149 Fällen, operiert im Intervallstadium oder als chronische Appendicitis war I Toter $=0,67$ Proz., nämlich ein Narkosetod durch Chloroform in I913. Seitdem ist diese Form der Narkose boi uns abgeschafft und ersetzt durch die Chloräthyläthernarkose.

\section{B. Frauen.}

\begin{tabular}{|c|c|c|c|c|c|c|}
\hline \multicolumn{2}{|c|}{ Alter } & & Akut & \multicolumn{2}{|c|}{ Gestorben Chronisch } & Gestorben \\
\hline$I-5$ & ahr & 7 & 7 & 3 & & \\
\hline $6-10$ & $, \quad:$ & 37 & 30 & 4 & 7 & \\
\hline $\mathrm{I} I-\mathrm{I} 5$ & , : : & 68 & 38 & I & 30 & \\
\hline I $6-20$ & " : & $\log$ & 54 & 2 & 55 & I \\
\hline $21-25$ & $, \quad:$ & 103 & $5^{2}$ & 3 & $5 \mathrm{I}$ & \\
\hline $26-30$ & $, \quad:$ & 52 & 34 & & 18 & \\
\hline $31-35$ & $, \quad:$ & $4 I$ & 22 & 3 & 19 & \\
\hline $36-40$ & ,. : & 26 & 10 & 1 & 10 & \\
\hline
\end{tabular}




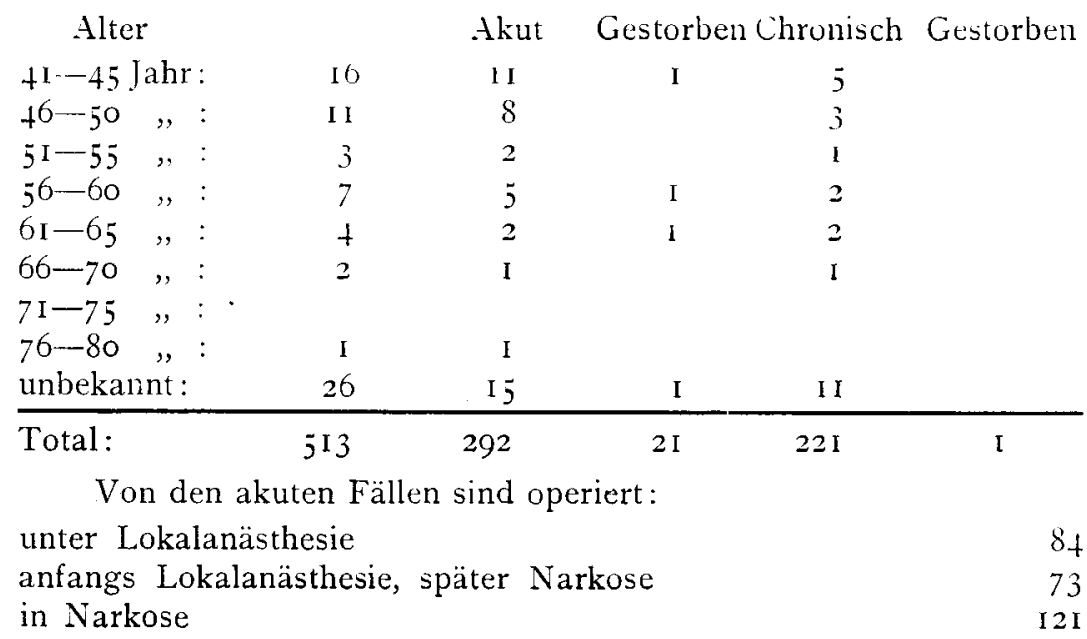

Appendicitis im Intervallstadium und chronische Appendicitis: unter Lokalanästhesie anfangs Lokalanästhesie, später Narkose $\quad 27$ in Narkose 94

Nur Abszeßöffnung und später nicht zurückgekommen für Radikaloperation: 5 unter Lokalanästhesie.

Drainage der Bauchhöhle bei diffuser Peritonitis ohne Appendektomie: 9 unter Lokalanästhesie.

Auf 292 akuten Fällen waren 2 I Tote $=7,3$ Proz., und zwar:

Peritonitis

Basalmeningitis

akute Nephritis

multiple spontane Darmfisteln

Pneumonie

Bronchopneumonien und Thymushyperplasie totaler Dünndarmvolvulus

Miliärtuberkulose

Von den 22 I chronischen Fällen starb I an Chloroformintoxikation nach der Narkose (in I9I I) $=0,45$ Proz.

In den Operationsprotokollen fand ich angegeben:

557 mal einen .,Wechselschnitt" nach Mac Burney.

383 , einen „Kulissenschnitt",

I2 ", eine mediane Laparotomie,

4, eine Leistenbruchinzision,

20 , eine Inzision längs dem rechten Rippenbogen,

25 , Flankendrainage bei diffuser Peritonitis,

I7 ," keine Angabe des Schnittes. 
Aufgenommen mit einem Infiltrat oder $A$ bszeB, der konservativ behandelt ist und später sekundäre Ektomie: $91 \mathrm{mit}$ oProz. Mor talität.

Aufgenommen mit einem $A b s z e B$, der geöfnet ist und später sckundäre Ektomie: 7 I mit o Proz. Mortalität.

Aufgenommen mit einem AbszeB, der geöffnet ist und später nicht zurückgekommen für Radikaloperation: $20 \mathrm{mit}$ o l'roz. Mortalität.

Sehr wichtig und lehrreich ist folgende Liste.

Akute Fälle hat man operiert:

innerhalb I Tag: 240 mit 7 Toten $=3$ Proz. Mortalität,

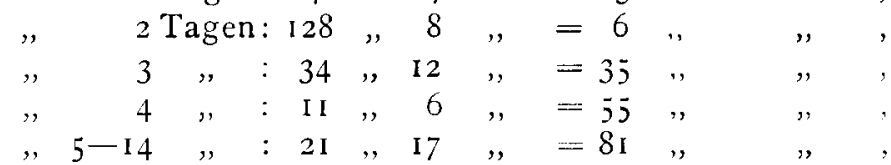

unbekannt nach wieviel Zeit: I4 mit keiner "

Die relativ hohe Sterbezahl bei den innerhalb des I. Tages operierten Fällen verlangt eine nähere Erklärung. Nicht weniger als vier der sieben Todesfälle hatten mit der Appendicitis nichts zu tun: Es waren:

ein totaler Dünndarmvolvulus, eine Woche nach der Operation, ein Fall von Miliärtuberkulose,

und zwei Kinder mit Bronchopneumonie und Thymushyperplasie, so daß nur 3 Fälle übrig bleiben, die zwar innerhalb 24 Stunden nach dem Beginn des Anfalles operiert wurden, jedoch alle drei schon eine ausgesprochene Peritonitis hatten; diese drei Fälle sind also in pathologisch-anatomischem Sinne nicht mehr als Frühfälle zu betrachten. Man darf hieraus die Folgerung zichen, da $B$ man diese Patienten eigentlich noch cher hätte operieren sollen; dieses ist also wicder eme Bekräftigung der Aufforderung die akute Appendicitis so bald wie möglich zu operieren.

In der obigen Liste sind also die akuten Fälle aufgenommen, die direkt nach der Aufnahme operiert sind. Teils waren dies Kranke, die im Frühstadium zu uns gekommen waren, teils waren es Fälle, die aufgenommen wurden mit mehr oder weniger ausgebreiteten peritonitischen Symptomen und die nun gleich operiert wurden, weil der Entzündungsprozeß keine Neigung hatte, sich zu lokalisieren.

Fanden wir jedoch eine lokale Peritonitis, war also ein Infiltrat oder Abszeß zu fühlen, dann wurde nach den oben erwähnten Indikationen gehandelt, d. h. man sah sich den Fall erst an 
und griff nur dann operativ ein, wenn der ProzeB progredient $\mathrm{zu}$ sein schien.

Wenn wir jetzt unsere Resultate betrachten, zeigt uns die oben erwähnte Statistik deutlich, ja deutlicher wie jedes gesprochene oder geschriebene Wort den großen Vorteil der Frühoperation. Ich werde nicht widersprechen, daß es auch akute Appendicitis gibt, die ohne Operation genesen kann, aber das weiß man niemals zuvor, und wenn die Entzündung einmal die Neigung zeigt sich auszubreiten, dann soll der Wiclersacher einer Fruihoperation sich vorhalten, daß er jeden Tag, den er länger wartet, die Aussicht auf Genesung für seine Kranken verringert. Ein Blick auf unsere Statistik zeigt uns, daß die Mortalität nach 3 Tagen schon romal größer ist als nach I Tag!

Obgleich $Z$ a a ijer der Meinung zugetan ist, da $B$ man nicht genug Wert darauf legen kann, um den akuten Anfall so bald wie nur möglich im allerersten Beginn der Krankheit zu operieren, bleibt jedoch seine abwartende Haltung gegenüber appendizitischen Infiltraten oder Abszessen bestehen angesichts unserer Resultate, die bei I 82 auf diese Weise behandelten Fällen eine Mortalität von o Proz. aufweisen.

In dieser Arbeit ist die Sterbeziffer der Peritonitisfälle nicht aufgenommen, da es nicht ganz genau zu umschreiben ist, was man eigentlich unter allgemeiner Bauchfellentzündung versteht. Finden wir bei der Operation einer nicht begrenzten Appendicitis mit peritonealen Symptomen Eiter über den Unterleib verbreitet, so sprechen wir von einer Peritonitis. Doch ist es keine allgemeine, sondern mehr eine ausgebreitete lokale Peritonitis; man darf wohl behaupten, daß die Fälle, wobei der ganze Unterleib in Entzündung begriffen ist und der Prozeß durch das Colon transversum nach oben zu begrenzt wird, die schwersten Formen der Peritonitis darstellen, die durch unsere Maßregeln noch günstig beeinflußt werden können. Ist dagegen wirklich die ganze Bauch. höhle krankhaft verändert, besteht also eine diffuse Peritonitis, so ist der Erfolg äuBerst gering.

$\mathrm{Da}$ ich aus den Operationsberichten nicht genau feststellen konnte, wie weit sich in jedem Fall die Peritonitis ausbreitete, ist 
es mir auch leider nicht möglich eine vergleichende Statistik über Peritonitis zu geben.

Auf Grund meiner Nachuntersuchungen meine ich nochmals auf folgende Konklusionen den Nachdruck legen zu müssen.

I. Es ist von großer Wichtigkeit, so bald wie möglich die akute Appendicitis zu diagnostizieren, damit man den Kranken im Frühstadium, wenn er die besten operativen Erfolge haben kann, nach dem Chirurgen verweisen kann.

II. Wenn es bei der Aufnalame in der Klinik deutlich ist, daß der Entzündungsprozeß sich schon nicht mehr allein auf den Wurmfortsatz beschränkt hat, jedoch schon eine lokale Peritonitis besteht in der Form eines Infiltrates, ist es sehr anzubefehlen, dies nicht als eine operative Indikation zu betrachten; man soll dann erst abwarten, ob die Krankheit die Neigung hat zurückzugehen oder ob eine deutliche Ausbreitung des Infiltrates mit Verschlimmerung der Symptome auftritt. In dem ersten Fall soll man den Kranken mit Bettruhe in Fowlerscher Lage, Diät usw. behandeln, bis das Infiltrat verschwunden ist und dann ungefähr 8 bis ıo Wochen später à froid operieren. Breitet die Entzündung sich aus, dann muß man operativ eingreifen.

III. Der Eingriff bei diesen schwerkranken Patienten soll eine einfache, kurzdauernde Operation unter Lokalanästhesie sein, d. h. eine Abszeßöffnung, wobei man nicht versuchen soll, den Wurmfortsatz zu entfernen, es sei denn, daß er sich direkt in der Wunde zeigt.

IV. Man verrichtet dann 8 bis Io Wochen später nach der Genesung der Abszeßinzision die Ektomie.

$V$. Wenn es sich bei der Aufnahme zeigt, daß schon eine allgemeine Peritonitis besteht und es uns bei der Palpation des Bauches deutlich wird, daß hier kein lokal begrenztes Infiltrat zu fühlen ist, dann wird sofort operiert, und zwar zur Bestreitung der schon vorhandenen drohenden, allgemeinen Peritonitis, wobei man versuchen wird den Wurmfortsatz, die Ursache der Krankheit, zu entfernen. Wenn der Zustand des Kranken die Appendektomie nicht rechtfertigt, beschränkt man sich darauf, durch Anlegung von Flankeninzisionen dem Eiter AbfluB zu geben.

Muß die Bauchhöhle nach der Ektomie drainiert werden, dann verwendet man am besten Cummidrains, die bis tief in das 
kleine Becken geführt werden, eventuell auch auf das Appendixbett gelegt werden. Tamponade mit Gazestreifen gibt Anlaß zu Verklebungen, wodurch man von der Drainage nicht viel Nutzen erwarten darf. Nur wenn man große Abszeßhöhlen schnell verkleinern will, kann die Tamponade mit Gazestreifen Nutzen haben.

Zur Verhütung einer Bauchwandinfektion soll man nicht $z \mathrm{u}$ sparsam sein mit der Drainage. Diese verlängert die Genesungsdauer nur für kurze Zeit, eine Bauchwandinfektion verzögert sie jedoch erheblich und gibt eher Anlaß zu Narbenbrïchen (Ber. tels mann, Wolff).

Deutet eine zunehmende Bauchauftreibung auf eine Darmparalyse hin, dann ist eine baldige Anlegung von Darmfisteln zu empfehlen. Man verringert hierdurch die Intoxikation des Kranken und die Ernährungsstörung der Darmwand. Am liebsten gebrauchen wir hierfür die Witzelsche Schrägfistel.

\section{Literaturverzeichnis.}

I. Bertelsmann, Soll im Intermediärstadium der akuten Appendicitis operiert werden? Deutsche Zeitschr. f. Chir. I913, Bd. 122.

2. Bullétins de l'Académie de Médecine. Paris, Jahrgr. 83, Bd. 82 .

3. D u rand, L'incision de la paroi abdominale dans l'appendicectomic it froid. Lyon, Méd. I920, No. 13, Ref. Zentralbl. f. Chir. I92 I, Nr. 3.

4. Eichhoff, Beiträge zur Behandlung appendizitischer Abszesse und Peritonitiden nach Rehn. Bruns' Beitr. 1919, Bd. II7, H. 2.

5. Eisendraht, Drainage of the abdominal wall in acute appendicitis. The journ. of the Americ. med. Ass., Dec. 1919.

6. Graser, Operative Behandlung der Appendicitis und Peritonitis. In: $\mathrm{Bier}, \mathrm{Br}$ a un und $\mathrm{K}$ ü $\mathrm{mmel}, \mathrm{I} 920, \mathrm{Bd} .3$.

7. Griep, Karl, Über die Behandlung der perityphlitischen Abszesse. Deutsche Zeitschr. f. Chir. J921, Bd. I62, S. 289.

8. Hagen, Zur Behandlung der akuten Appendicitis. Münchner med. Wochenschr. 1919, Nr. 49.

9. Krecke, A., Beitrag zur Behandlung der akuten Appendicitis, insbesondere bei der umschriebenen Abszeßbildung. Nünchner med. Wochenschr. 1919, Nr. 37 .

10. $\mathrm{Krogius}$. In $\mathrm{B}$ ier, $\mathrm{Br}$ a un und $\mathrm{K}$ üm $\mathrm{m}$ el. Chirurgische Operationslehre Bd. 3 .

I I. L a n $z, O$, Welk is het gunstigste oogenblik roor chirurgisch ingripen bij appendicitis? Handelingen van het elfde Nederlandsch Natuur-en Geneeskundig Congres, p. 365. 
12. Morris. Robert T. Ref. Zentrabl. f. Chir. I92I. Nr. 3.

13. Riediger. Horst. Die Operationserfolge ron 98 appendizitischen Abszessen. Ref. Zentralbl. f. Chir. 1920, Nr. 32.

14. Rulison. Drainage in Appendicitis. Annals of surg. 1919, Vol. LXX, No. 6 .

15. Steiger, l'ber dic Behandlung der Blinddamentzündung. Berliner klin. Wochenschr. 1920. Nr. 2.

16. So h n, A., Ober die Spülung des kleinen Beckens bei der Behandlung der diffusen eitrigen Peritonitis. Bruns' Beitr. Bd. I2I, H. I, S. I9I.

I7. Stutzin, J., Experimentelle und klinische Beiträge zur Drainage und Spülbehandlungr entzündlicher Erkrankungen der Bauchhöhlc. Deutsche Zeitschr. f. Chir. 1919. Bd. 149.

18. Westerman, C. W. J., Uber die Anwendung des Dauermagenhebers bei der Nachbehandlung schwercr Peritonitisfälle. Zentralbl. $\dot{f}$. Chir. 1910, Nr. 10. S. 350 .

[4. WjJms. Die Behandlung schwerer Fäle ron Peritonitis beim Ilann durch Drainage des Douglas nach dem Mastdarm zu. Deutsche Zeitschr. f. Chir. I9I7, Bd. I39.

20. Wolff, Ernst, Zur Behandlung der appendizitischen Abszesse. Bruns' Beitr. Bd. I I , H. 2. S. 263.

zi. Zur l' erth, Die Technik der Blinddarmoperation. Ref. Zentralbl. f. Chir. 1920. Nr. 42 